\title{
Distribución y Conectividad Estructural de Bosques de Polylepis Mediante el Uso de Imágenes Satelitales en Tres Áreas de Conservación Privada en el Valle del Vilcanota - Cusco
}

\section{Distribution and Structural Connectivity of Polylepis Forests through the Use of Satellite Images in Three Areas of Private Conservation in the Vilcanota Valley - \\ Cusco}

\section{Katy V. Rondinel Mendoza}

Facultad de Ciencias - Escuela Profesional de Biología - Universidad Nacional de San Antonio Abad del Cusco.krondinel@gmail.com

\begin{abstract}
Resumen
El presente trabajo se realizó en tres Áreas de Conservación Privada (ACP) Mantanay, ACP Abra Málaga Thastayoq Royal Cinclodes y ACP Hatun Queuña Quishuarani - Valle delVilcanota - Cusco, la finalidad fue conocer la distribución y conectividad estructural de bosques de Polylepis mediante el uso de imágenes satelitales. Para dicho fin, se establecieron parámetros que determinen el mejor desarrollo de las futuras plántulas, dichos parámetros fueron: altitud entre 4100 a $4550 \mathrm{~m}$.; pendiente 21 a 45 grados, priorizándose áreas en dirección general sur (sureste, sur, suroeste y oeste) y rango de radiación entre 5.5 a $7.0 \mathrm{KWh} / \mathrm{m}^{2}$. La distribución dio a conocer la dispersión de parches, disponibilidad de la matriz y el hábitat para la reforestación, estas características ayudaron a determinar la conectividad estructural ecológica con el software Conefor Sensinode 2.6, cuyos índices denotaron el buen incremento de las masas arbóreas y la matriz. Asi mismo, las especies encontradas en cada ACP fueron: Polylepis racemosa y P. subsericans., para el ACP Mantanay y P. sericea y P. pepei., para las ACPs Abra Málaga y Quishuarani., con los datos obtenidos se puede ayudar a las comunidades campesinas en el manejo de sus servicios ecosistémicos por ende la eficacia de los programas de manejo, uso y conservación de bosques depende del buen uso que le den al hábitat.
\end{abstract}

Palabras clave: Conectividad, distribución, imágenes satelitales, matriz, conefor Sensinode.

\begin{abstract}
This present work, has been made in three Private Conservation Areas: ACP Mantanay, ACP Abra Málaga Thastayoq Royal Cinclodes and ACP Hatun Queuña Quishuarani in the Vilcanota Valley - Cusco, the purpose was to determine the distribution and structural connectivity the forests of Polylepis using satellite images. For that purpose, were established parameters that favoring the development of future seedlings like: altitude between 4100-4550 m.; Slope 21-45 degrees, giving priority areas with general direction south (southeast, south, southwest and west) and range of radiation between 5.5 to $7.0 \mathrm{KWh} / \mathrm{m}^{2}$. The distribution show the dispersion patch, availability of the matrix and habitat for reforestation, these features helped to determine the ecological structural connectivity with Conefor Sensinode 2.6 software, whose indices have denoted the good increase forested areas and the matrix. Of the species found in each ACP were: Polylepis racemosa and P. subsericans for the ACP Mantanay and P. sericea and P. pepei for the ACPs Abra Málaga and Quishuarani. This data obtained can help the peasant communities in the management of their ecosystem services, so the effectiveness of forest management, use and conservation programs depends on how well they use the habitat.
\end{abstract}

KeyWords: Connectivity, distribution, satellite images, matrix, Conefor Sensinode. 


\section{Introducción}

Los bosques Altoandinos de la Cordillera de los Andes con altitudes por encima de los 3.000 $\mathrm{m}$ son escasos, debido a la fragmentación de su hábitat por factores antropogénicos por lo que se encuentran localizados denominándose relictos de bosque y se encuentran rodeados de vegetación circundante correspondiente a zonas agrícolas y pastizales. Estos bosques están restringidos a laderas rocosas o quebradas dominadas por especies del género Polylepis, que se extienden desdeVenezuela hasta el norte de Chile y Argentina. Incluye cerca de 27 especies (Simpson 1979; Cabido \& Acosta 1985; Kessler 1995, Romoleroux 1996; Mendoza 2005, 2010; Bosch 2012). Estos bosques se encuentran asociados a especies leñosas como Budleja coriacea, Myrcianthes oreophylla, Gynoxys aff. Nitida, Gynoxys longifolia. El rol de dichos bosques en la ecología altoandina es ser fuente de recursos para la población local (Lovejoy et al. 1986; Galiano 1990; Murcia 1995, Gentry 1995; Fjeldsa \& Kessler 1996; Laurance \& Bierregaard 1997; Kessler 2006). Los bosques de Polylepis (queuñal) de la Cordillera del Vilcanota y Urubamba, son ecosistemas con fauna y flora única especialista y endémica (Servat, 2002).

El presente estudio tiene como objetivo determinar la distribución y conectividad estructural de bosques de Polylepis mediante el uso de imágenes satelitales en tres Áreas de Conservación Privada en el Valle del Vilcanota - Cusco, mediante el análisis de tres factores ambientales: la altitud, la pendiente, la exposición solar y el uso de imágenes satelitales para determinar la conectividad estructural basada en la distancia y continuidad entre teselas de bosque utilizada como herramienta de planificación territorial, cuyos parámetros permitirán determinar áreas adecuadas para el buen desarrollo de las futuras plántulas de Polylepis.

\section{Material y métodos}

\section{Área de Estudio}

El presente estudio fue realizado en la Coordillera del Vilcanota - Urubamba, a dos horas de la ciudad del Cusco. Según Brack 1986, ecológicamente, las áreas de estudio se encuentran situada en la eco región de Puna semi Húmeda (3 800 m. a 5200 m de altitud).

\section{Métodos}

\section{Obtención y preparación de imágenes de satélite:}

Se trabajó con dos imágenes satelitales Landsat: Landsat 7 TM (Thematic Mapper) para la clasificación preliminar y Landsat ETM+ (Enhanced Themapic Mapper Plus) para la clasificación final. Estas imágenes se obtuvieron del Servicio Geológico de los Estados Unidos (USGS), correspondientes al mes de Julio del 2010, también se obtuvo el DEM (Mapa de Elevación Digital) proporcionado por el MINAM-Perú proyectándose a la data WGS 84 ZONA 19, procesadas en el software Esri Arcgis v 10.1 en una escala de 1:50000, en este software se cargaron los datos obtenidos por GPS en coordenadas UTM para la ubicación y delimitación del área de cada bosque evaluado. Se compuso en tres bandas ( 5 red, 4 green y 3 blue según RGB) que mejor reflejaran los bosques de Polylepis, resultando una resolución final de 30m x 30m, y, como material de apoyo se utilizó una imagen de satélite del programa Google Earth de las zonas de estudio del año 2013 para una mejor visualización a nivel de individuo. Además se utilizaron fotografías aéreas que datan del año 1956, adquiridas en la DIRAF (Dirección de Aerofotografía de la Fuerza Aérea del Perú - FAP). 
correspondientes a las zonas de estudio para hacer una comparación temporal de distribución de Polylepis. Las fotografías aéreas, corresponden a los siguientes proyectos: 8485 A4 - Abra Málaga y 8485 A4 - Mantanay.

Cabe señalar que la fotografía aérea para el área de estudio "Quishuarani" en Calca, no se encuentra editada aún por la DIRAF debido a las condiciones climáticas adversas y al elevado costo de sobrevuelos y mantenimiento de equipos fotográficos.

\subsection{Ubicación, mapeo y análisis de} bosques.

Se realizaron salidas preliminares para mapear las zonas de estudio mediante el uso del track alrededor de las masas arbóreas y en algunos casos la toma de puntos georeferenciales, que ayudaron a definir los elementos del paisaje (bosque, pasto, roca, glaciar, etc.), además el apoyo del conocimiento de los pobladores de las zonas fue preciso en la definición de los mismos en campo.

Cuadro 1: Ubicación geográfica de las ACPs - ámbito de estudio.

\begin{tabular}{|c|l|l|l|l|l|l|}
\hline $\mathbf{N}^{\circ}$ & ACP & Provincia & Distrito & Comunidad & Altitud & Coordenadas \\
\hline $\mathbf{1}$ & $\begin{array}{l}\text { Abra Málaga } \\
\text { Thastayoc Royal } \\
\text { Cinclodes }\end{array}$ & Urubamba & Ollantaytambo & $\begin{array}{l}\text { Abra Málaga } \\
\text { Thastayoc }\end{array}$ & $3950-4250$ & $18 \mathrm{~L} 8545000793000$ \\
\hline $\mathbf{2}$ & $\begin{array}{l}\text { Hatun Queuña } \\
\text { Quishuarani } \\
\text { Ccollana }\end{array}$ & Calca & Lares & $\begin{array}{l}\text { Quishuarani } \\
\text { Ccollana }\end{array}$ & $3950-4850$ & $18 \mathrm{~L} 854400820000$ \\
\hline $\mathbf{3}$ & Mantanay & Urubamba & Urubamba & $\begin{array}{l}\text { San Isidro } \\
\text { Cusibamba }\end{array}$ & $3050-2850$ & $18 \mathrm{~L} 8535000807000$ \\
\hline
\end{tabular}

Cabe señalar que la fotografía aérea para el área de estudio "Quishuarani” en Calca, no se encuentra editada aún por la DIRAF debido a las condiciones climáticas adversas y al elevado costo de sobrevuelos y mantenimiento de equipos fotográficos.

\subsection{Ubicación, mapeo y análisis de} bosquesSe realizaron salidas preliminares para mapear las zonas de estudio mediante el uso del track alrededor de las masas arbóreas y en algunos casos la toma de puntos georeferenciales, que ayudaron a definir los elementos del paisaje (bosque, pasto, roca, glaciar, etc.), además el apoyo del conocimiento de los pobladores de las zonas fue preciso en la definición de los mismos en campo.
Se trabajó con las siguientes especies de Polylepis para las diferentes ACPs: Polylepis racemosa R\&P, P. besseri Hieron y P. subsericans J.F.Macbr., para el ACP Mantanay; P. serícea Wedd. y P. pepei B.B. Simpson para las ACPs Abra MálagaTRC y Quishuarani.

\section{Elaboración de los Factores a nivel de paisaje.}

El procesamiento de los datos de campo consistió en la digitalización y generación de los polígonos de bosques de Polylepis cuya ubicación y extensión otorgaron los fundamentos para poder relacionarlos con los factores a nivel de paisaje (altitud, orientación solar y pendiente), y así identificar la fragmentación de los bosques, a su vez generar los mapas correspondientes mediante el uso del MDT y vectorizar cada una de las capas raster, es decir el cálculo de las áreas de cada- 
-polígono. Los rangos establecidos para los diferentes factores fueron dados según una comparación con los rangos preestablecidos por parte del "Mapa de Pendientes para el Cusco del 2012 - GORE Cusco, SENAMHI, el Ministerio de Energía y Minas y por el Fondo Mundial para el Medio Ambiente (GEFPNUD) en su documento de "Atlas de Energía Solar del Perú".

\section{Determinación de la conectividad}

\section{entre teselas de bosque.}

\section{Análisis de la conectividad ecológica.}

Para fines de este estudio se toma en cuenta las medidas de la conectividad estructural, que utilizan un algoritmo basado en un ráster que calcula la distancia efectiva basada en la dispersión de una determinada especie (coste acumulado para el movimiento) como lo indica Adriaensen et al.(2003). Para dicho análisis se utilizó el Conefor Sensinode, un programa informático que permite medir la contribución de cada tesela de hábitat para el mantenimiento o posible mejora de la conectividad ecológica (Pascual- Hortal y Saura 2006, Saura y Rubio 2009).

\section{Análisis de Índices}

a.- Índice de la Probabilidad de la Conectividad (PC):

La PC se define como la probabilidad de que dos puntos colocados aleatoriamente en el paisaje en áreas de un hábitat sean accesibles entre sí. Con valores de $(0$ a 1$)$ donde 0 representa la conexión más cercana efectiva y 1 la conexión más lejana (Saura y Pascual-Hortal, 2007), expresado en porcentaje y viene dada por:

$$
P C=\frac{\sum_{i=1}^{n} \sum_{j=1}^{n} a_{i} \cdot a_{j} \cdot p_{i j}^{*}}{A_{L}^{2}}
$$

\section{Dónde:}

n: número de parches de hábitat existentes en el paisaje tanto para i como j

$\mathbf{a}_{\mathrm{i}}, \mathbf{a}_{\mathrm{j}}$ : área de cada parche de hábitat.

$\mathbf{A}_{\mathrm{L}}$ :área total del paisaje (tanto del hábitat y no hábitat).

$\mathbf{p}{ }^{*}$ ij:probabilidad de dispersión entre los parches i y j (Bunn et al, 2000; Fu et al, 2010.), es decir la probabilidad máxima del producto, el valor máximo de todas las posibles vías de conexión entre los parches i y j que van de ( $0 \mathrm{a}$ 1), tomando el valor máximo de 1 .

\section{b.- Índice Integral de Conectividad (CII):}

Esta medida utiliza la distancia topológica (dij) entre parches i y j (distancia más conexa entre ambos parches). Es un índice con valores desde 0 hasta 1 , incrementándose ante una mayor conectividad expresado en porcentaje (Pascual-Hortal y Saura, 2006; Saura y PascualHortal, 2007).

$$
\text { IIC }=\frac{\sum_{i=1}^{n} \sum_{j=1}^{n} a_{i} \cdot a_{j} \cdot\left(1 /\left(1+d_{i j}\right)\right)}{A_{L}^{2}}
$$

\section{Dónde:}

n: número de parches de hábitat existentes en el paisaje tanto para i como j

$\mathbf{a}_{\mathrm{i}}, \mathbf{a}_{\mathrm{j}}$ : área de cada parche de hábitat.

$\mathbf{A}_{\mathrm{L}}$ : área total del paisaje (tanto del hábitat y no hábitat).

$\mathbf{d}_{\mathrm{ij}}$ : distancia topológica (dij) entre parches i y j (distancia más conexa entre ambos parches). 


\section{c.- Índice del Área Conexa Equivalente (ACE)}

Se define como la superficie plenamente conexa que le corresponda la misma probabilidad de conectarse con otras teselas o parches. La ventaja es, que permite la comparación de manera relativa del cambio de superficie boscosa que beneficia a la conectividad y el intercambio de flujos ecológicos. (Pascual-Hortal y Saura, 2006; Saura y Pascual-Hortal, 2007).

$$
A C E=\sqrt{\sum_{i=1}^{n} \sum_{j=1}^{n} a_{i} \cdot a_{j} \cdot p_{i j}^{*}}
$$

\section{Dónde:}

n: número de parches de hábitat existentes en el paisaje tanto para i como j

$\mathbf{a}_{\mathbf{i}}, \mathbf{a}_{\mathbf{j}}$ : área de cada parche de hábitat.

$\mathbf{A}_{\mathbf{L}}$ : área total del paisaje (tanto del hábitat y no hábitat).

p* ${ }_{\mathrm{ij}}$ : probabilidad de dispersión entre los parches i y j (Bunn et al, 2000; Fu et al. 2010.), es decir la probabilidad máxima del producto, el valor máximo de todas las posibles vías de conexión entre los parches i y j que van de $(0 \mathrm{a}$ 1), tomando el valor máximo de 1 .

\section{Resultados}

Análisis espacial de los parches de bosque de Polylepis.

Se contó un total de 121 polígonos o parches de bosque de Polylepis haciendo un total de $232 \mathrm{Ha}$. de extensión. La zona de estudio con mayor cantidad de parches y mayor extensión de bosques es Mantanay (80 parches en 140 Has.); le sigue Quishuarani (10 parches en 50 Has.) y por último Abra Málaga Tasthayoc (31 parches en 42 Has.) (Figura 1).

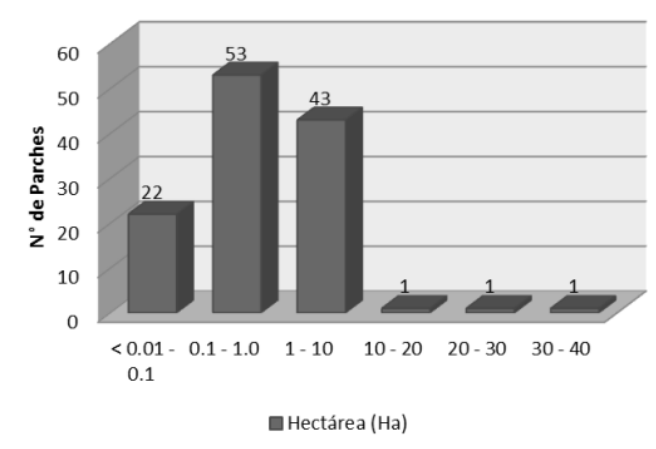

Figura 1: Número de parches de Polylepis según Rango deárea.

\section{Altitud}

Este factor ayuda a determinar la localización altitudinal de bosques naturales de Polylepis (Kessler 2006) (Figura 2).

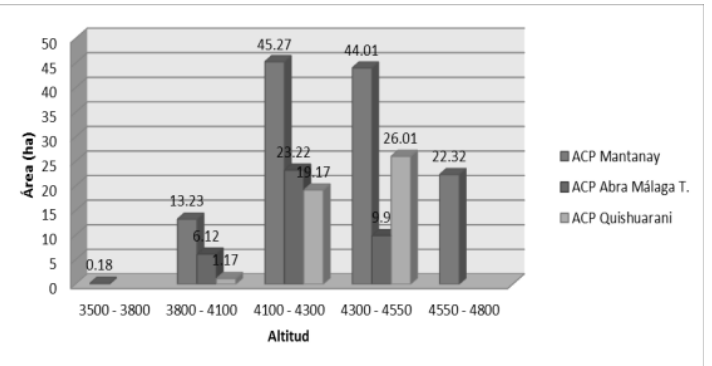

ACPs según rangos de altitud.

Por lo tanto para fines de este estudio se establece los parámetros ideales de altitud para la reforestación de bosques de Polylepis con un rango altitudinal de 4100 a $4550 \mathrm{~m}$. como la franja más apta, por encontrarse allí la más alta concentración de bosques naturales.

\section{Pendiente}

En las zonas de estudio se da una variabilidad de pendientes. Los datos obtenidos en este estudio, muestra que en el mapa de pendientes existen zonas completamente planas ( 0 grados de pendiente) hasta cuestas de casi 60 grados de pendiente: aunque es importante mencionar que existen paredones cercanos a las zonas de estudio. (Figura 3). 


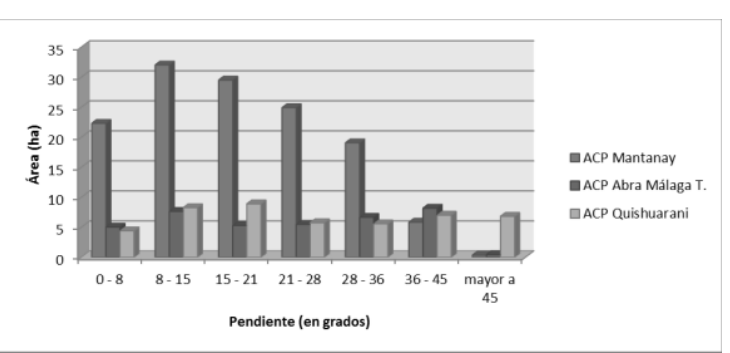

Figura 3: Total de hectáreas según rangos de pendiente en las tres ACPs.

Por lo tanto para fines de este estudio se establece los rangos de pendiente entre 21 a 45 grados.

\section{Radiación Solar y Orientación Respecto al Sol.}

Se ha divido el compás en dos direcciones generales, sur y norte. (Figura 4 y Tabla 1). La ubicación de los bosques marca una tendencia a estar expuestos a laderas que están orientadas al sur en un 53\%, mientras que al norte hay 47 $\%$ de los bosques.

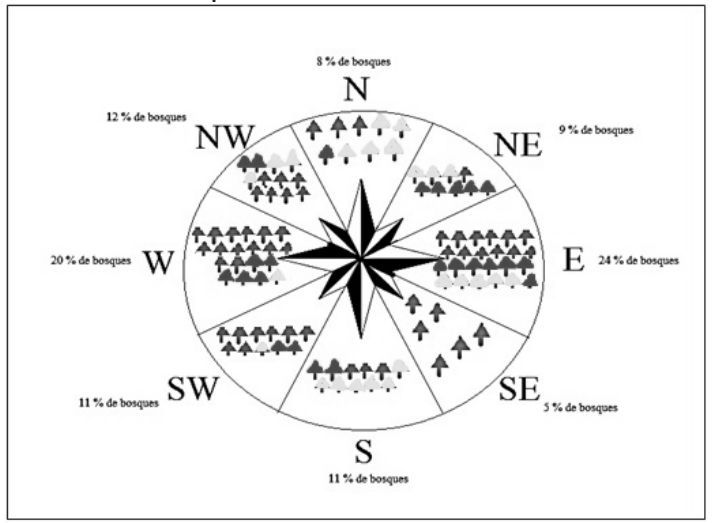

Figura 4: Diagrama de orientación del total de bosques en porcentaje según exposición solar para las tres ACPs

\section{Orientación respecto al Sol para cada ACP.}

Al analizar cada una de las áreas de estudio para las tres ACPs de acuerdo a su orientación según la exposición solar, se destaca que hay una gran variante con relación a que direcciones se dirigen más los parches de bosques para cada área (Tabla 1).

Tabla 1: Total de hectáreas de bosque expresadas en porcentaje en la orientación respecto al sol para cada ACP.

\begin{tabular}{|l|r|r|r|r|r|r|r|r|}
\hline & \multicolumn{1}{|c|}{ Norte } & \multicolumn{1}{c|}{ Sur } & \multicolumn{1}{c|}{ Oeste } & \multicolumn{1}{c|}{ Este } & Nor-Oeste & Nor-Este & Sur-Oeste & Sur-Este \\
\hline ACP Mantanay & 7.78 & 8.4 & 39.8 & 24.9 & 20.7 & 1.35 & 25.6 & 11.47 \\
\hline ACP Abra Málaga T. & 1.93 & 2.48 & 5.06 & 17.19 & 3.26 & 11.09 & 0.99 & 0 \\
\hline ACP Quishuarani & 8.4 & 15.48 & 0.5 & 13.41 & 4.81 & 7.31 & 0.09 & 0 \\
\hline TOTAL en ha & 18.11 & 26.36 & 45.36 & 55.5 & 28.77 & 19.75 & 26.68 & 11.47 \\
\hline Total en \% & 7.81 & 11.36 & 19.55 & 23.92 & 12.4 & 8.51 & 11.5 & 4.94 \\
\hline
\end{tabular}

\section{Radiación solar para cada ACP.}

En la tabla 2 se observa que los rangos por debajo de $7.0 \mathrm{KWh} / \mathrm{m}^{2}$ y por encima de 5.5 $\mathrm{KWh} / \mathrm{m}^{2}$, la cantidad de parches de bosque en porcentaje es relativamente mayor en orientación sur que al norte.

Tabla 2: Tamaño de bosque en hectáreas de cada ACP según mayor o menor radiación solar en direcciones generales NORTE y SUR para los meses febrero, mayo y noviembre.

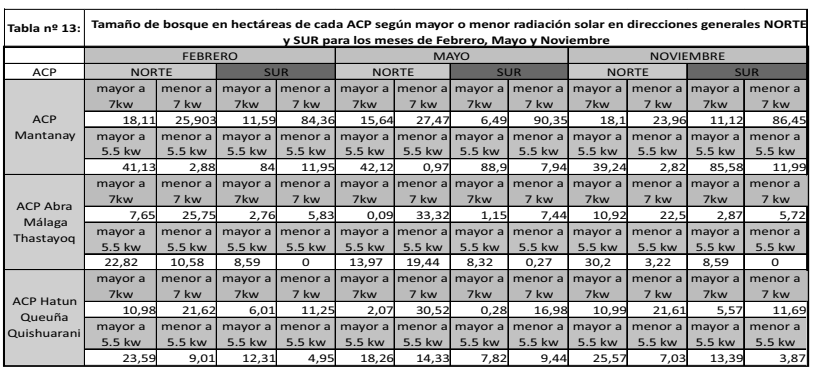




\section{Determinación de la conectividad} ecológica estructural entre teselas de bosque de polylepis.

a.- Índice de Probabilidad de la Conectividad (PC).

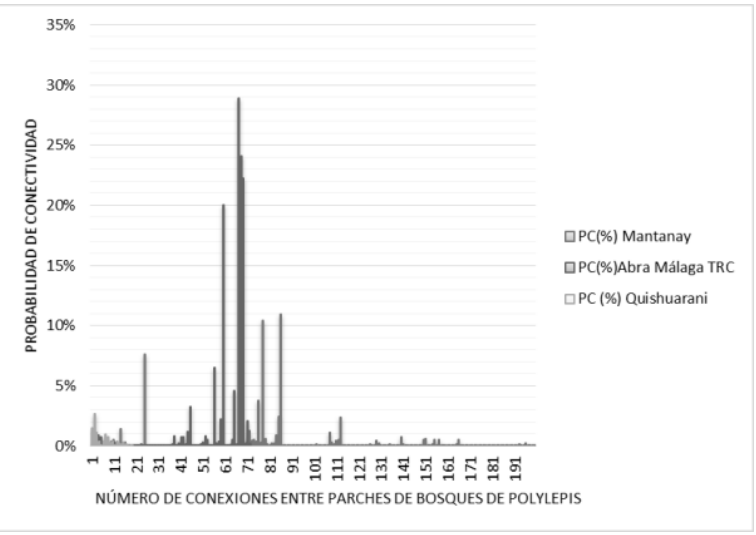

Figura 5: PC para los parches de las ACPs

El índice de PC medida en porcentaje según el número de conexiones entre parches de bosque de Polylepis para las tres ACPs, presenta valores de probabilidad de conexión cercanos a "0" lo que significa que para los tamaño de estos bosques la conectividad es alta debido a que los parches se encuentran cerca y conectados por los flujos ecológicos (ciclo del agua, ciclos biogeoquímicos, flujo de energía y la dinámica de las comunidades), otros se alejan por las distancias que son relativamente más largas a través de las masas boscosas.

\section{b.- Índice Integral de Conectividad (CII).}

El CII para las tres ACPs, presentan un índice integral de hasta 1 en algunos enlaces, lo que indica que la conectividad entre parches de bosque se desarrolla a medida que los mismos crecen y los flujos ecológicos son más constantes.

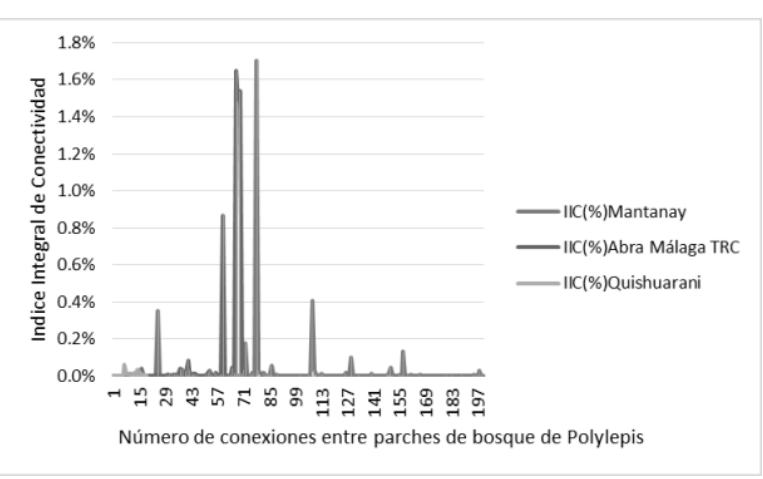

Figura 6: CII para los parches de las ACPs

\section{c.- Índice del Área Conexa Equivalente (ACE).}

Los índices del ACE para los casos expuestos llegan a tener áreas conexas equivalentes desde 2ha hasta más de 160 ha lo que significa que el crecimiento de las masas arbóreas se ven favorecidas por el desarrollo de las teselas de hábitat forestal.

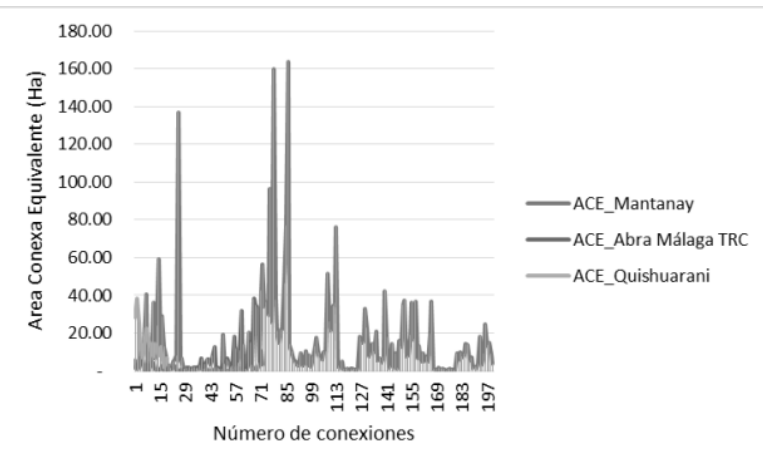

Figura 7: ACE para los parches de las ACPs

\section{Áreas con potencial para albergar plantaciones de Polylepis}

De los parámetros establecidos para los diferentes factores analizados, se obtiene un producto "mapa base" donde se muestran las- 
áreas a reforestar y que ayuda a ver mejor la distribución y conectividad de los parches basándose en la influencia que ejercen los diferentes factores y procesos ecológicos así como la dispersión de las especies.

\section{Discusiones}

La fragmentación de estos bosques a lo largo del tiempo en dichas zonas han generado cambios en el hábitat incluso disminuyendo la conectividad entre parches conformando ámbitos dispares como se menciona en estudios relacionados en Murcia 1995, Laurance y Bierregaard 1997, Lovejoy et al. 1986, la creación de transiciones abruptas conformando ambientes dispares (p. e. bosques y cultivos).

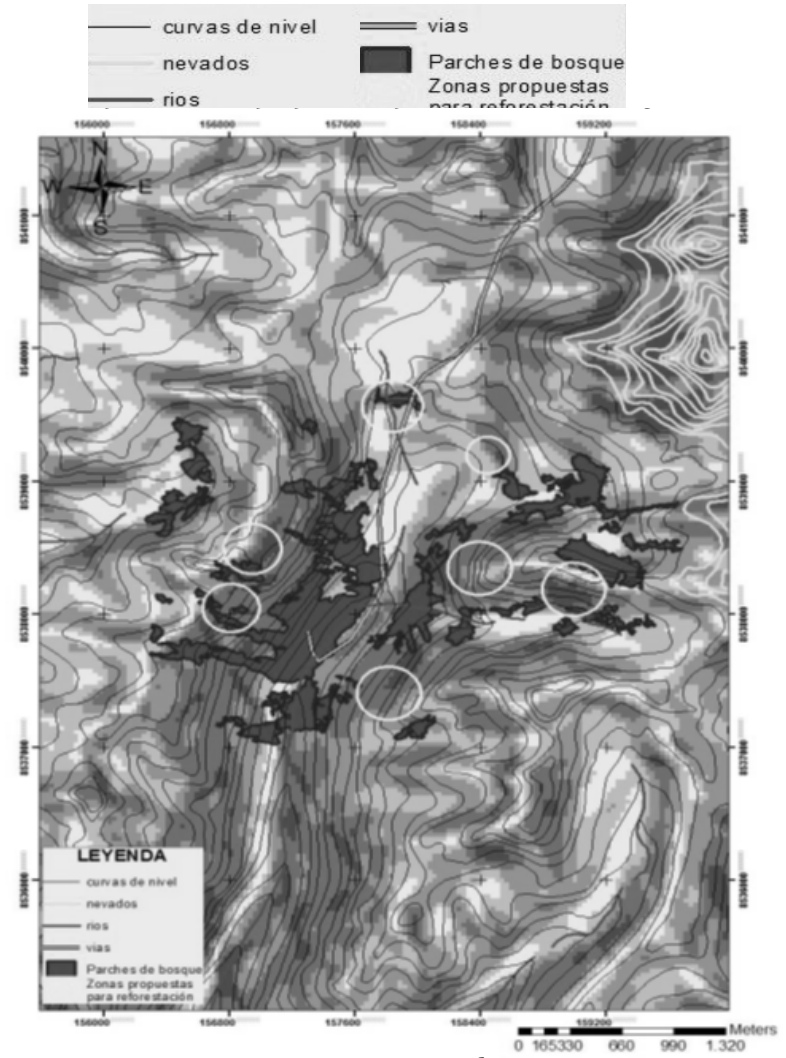

Figura 8: Zonas propuestas para la ACP Mantanay

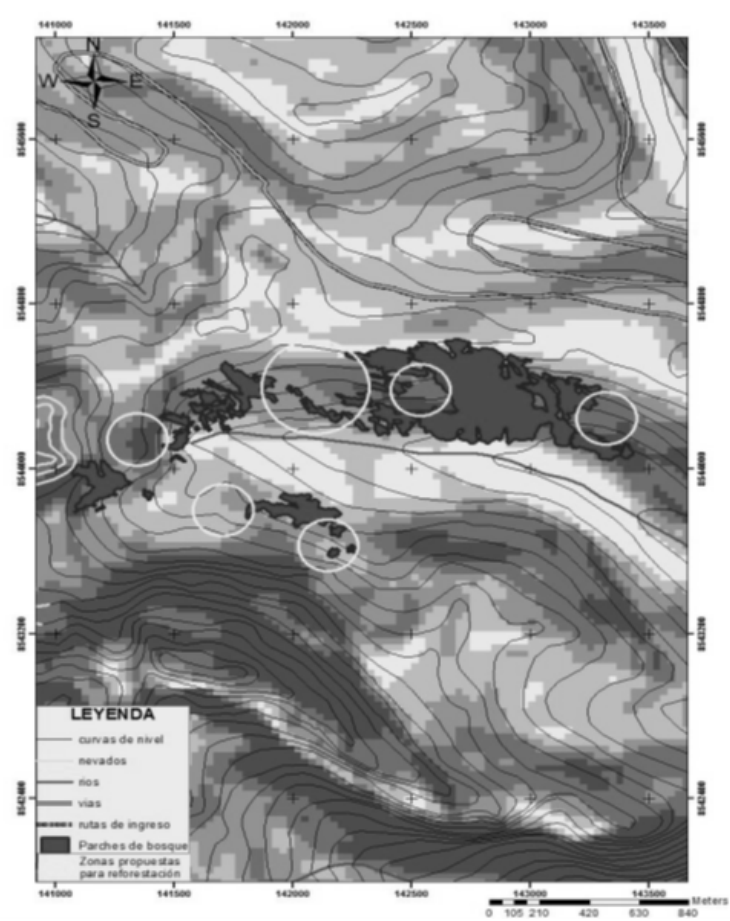

Figura 9. Zonas propuestas para la ACP Abra Málaga

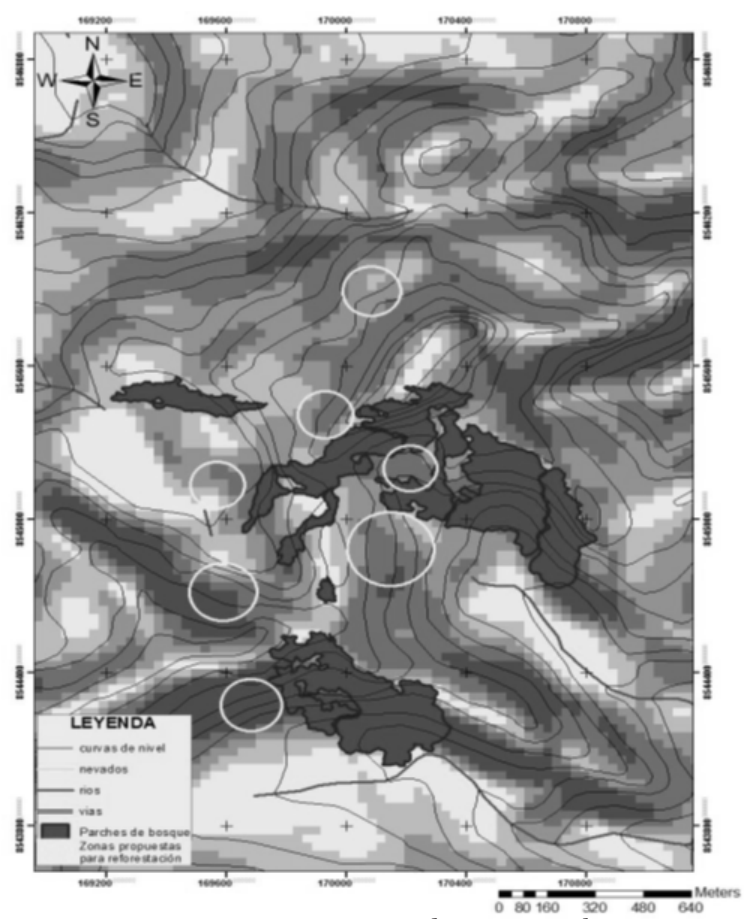

Figura 10. Zonas propuestas para la ACP Quishuarani 
Estas transiciones o bordes, generan cambios en las condiciones microambientales en el bosque contiguo, a estos cambios entre los hábitats adyacentes, se les ha denominado efecto de borde.

Con todos los resultados obtenidos para lograr los rangos más acordes de los factores ecológicos tomados en cuenta en este estudio con la finalidad de establecer científicamente los parámetros ambientales que favorecen el crecimiento y desarrollo de las futuras plántulas, como son: para la altitud entre 4100 a $4550 \mathrm{~m}$. ; pendiente 21 a 45 grados y la exposición solar se prioriza las áreas orientadas en dirección general sur (sureste, sur, suroeste y oeste), con el rango de radiación entre 5.5 a $7.0 \mathrm{KWh} / \mathrm{m}^{2}$. Esto no impide que no haya desarrollo por encima o debajo de los valores establecidos sino que se encuentran limitados sean por barreras geográficas naturales como por ejemplo roca maciza, nevados o barreras antropogénicas como la población, actividades de agricultura y ganadería, los cuales hacen uso igual de la matriz de estos ecosistemas (pajonal), dicha afirmación se corrobora en los estudios realizados por Galiano en 1994 en otras zonas altoandinas como en el Valle de Urubamba (Cusco) y en Puno, la presencia de los bosques de Polylepis tanto arbustos como árboles, se ubican a elevaciones entre 3,400$4800 \mathrm{~m}$, distribuidas en poblaciones aisladas, rodeadas por vegetación de la "puna”. Algunas especies de Polylepis crecen por encima de la "Línea de los Árboles" como en el Valle de Urubamba se encuentran sobre los $4800 \mathrm{~m}$. y a 5200 m en el Dpto. de Puno. En relación a la exposición solar muchos de los parches de bosque de las áreas estudiadas están condicionados a las horas luz expuestas y la cantidad de radiación recibida, lo mismo sucede en los bosques del Altiplano puneño,- -que está condicionada no solo por la cantidad de insolación recibida por las laderas donde se ubican, sino también por la dirección de los vientos predominantes que 'reacomodan' los efectos de la insolación, según lo mencionaYallico (1992).

La distribución de los bosques en la Cordillera del Vilcanota, se da en laderas y quebradas rocosas de manera aislada, las cuales son calentadas durante los días soleados además del ascenso desde el fondo de valle del aire caliente que atraviesa las estrechas quebradas y laderas y al llegar a las cumbres chocan con el aire frío, se condensan generando humedad, aún en áreas áridas, favoreciendo el desarrollo de estas poblaciones. Por otro lado, la inclinación del suelo y la presencia de abundante musgo asociado a hierbas, permiten la retención del agua de lluvia entre las raíces de los árboles, las que favorecen su crecimiento como lo menciona Tupayachi (2004). Esto permite afirmar que tanto la radiación como la pendiente en las zonas de estudio son factores que influyen en el desarrollo de las masas arbóreas de Polylepis incluso de otras especies asociadas a ellos.

En la Cordillera Blanca, en el departamento de Ancash, con el mismo propósito de estudio (Dourojeanni 2008), además menciona que la matriz de estos ecosistemas le da soporte a los elementos del paisaje presentes en las zonas de estudio. Para este estudio, la matriz de las zonas de estudio es la más apta para la restauración y reforestación de los bosques de Polylepis puesto que ninguno de los otros elementos como bofedales, zonas agrícolas, glaciares, etc no tienen las condiciones para albergar plantaciones.

En relación a la conectividad estructural establecida en base a los índices analizados, permite afirmar que entre los parches de- 
-bosque, dicha relación es alta debido a que los parches de bosque se encuentran cercanos entre si además la influencia que ejercen sobre ellos los diferentes factores y procesos ecológicos así como la dispersión de las especies. Igualmente se afirman en los trabajos de Dourojeanni 2008 para Perú y Saura 2011 en España, ambos coinciden en que la conectividad entre parches o teselas de bosque se da a medida que la cercanía entre ellos sea mayor y por ende los procesos ecológicos son más influyentes sobre las masas arbóreas.

Otro significado de todo este trabajo es: los bosques de Polylepis están en peligro de extinción y su equilibrio depende del uso sostenible de la población en general. Como complemento a todo lo desarrollado anteriormente, se hizo una comparación espacio-temporal de los bosques de las zonas de estudio para determinar cómo es que los bosques variaron con el tiempo. Las fotografías aéreas del año 1956 se comparó con imágenes de alta definición extraída del Google Earth del año 2014; es decir 57 años de diferencia entre una y otra imagen por lo que se puede asegurar que los bosques en toda la zona de estudio han cambiado poco, por lo que se tiene que hasta la fecha para el ACP Mantanay se perdió 73.09 ha de bosque de 213 ha registradas para ese año y el ACP Abra Málaga Thastayoq Royal Cinclodes perdió 9.5 ha de bosque de 52 ha registradas para ese año, no se obtuvo imagen de la zona de Quishuarani porque la DIRAF no presenta dichos reportes para la época.

\section{Agradecimientos}

A mi asesor Blgo. Percy Yanque y co-asesor MSc. Ronal Rojas por el apoyo, por brindarme sus conocimientos y consejos en la realización de este proyecto. A mis docentes Mgt. Luciano-
-Cruz, M.Sc. Washington Galiano, Percy Nuñez por sus consejos, Al Herbario Vargas CUZ de la Facultad de Ciencias Biológicas; a ECOAN como nexo con las comunidades campesinas a las cuales extiendo mis agradecimientos por el alojamiento. A la DIRAF y a cada uno de mis compañeros de trabajo por las experiencias y momentos compartidos.

\section{Literatura citada}

Adriaensen, F., Chardon, P., DeBlust, Swinnen, S., Villalba, H., Gulinck, \& E. Matthysen. 2003. The application of 'least-cost' modelling as a functional landscape model. Landscape and Urban Planning 64:233-247

Cabido, M. \& Acosta, A. 1985. Estudio Fitosociológico en bosque de Polylepis australis Bitter: ("Tabaquillo") en las sierras de Córdoba. Argentina. Documents phytosociologiques. N.S.Vol. IX: 365-400.

Dourojeanni, P. 2008. Distribución Y Conectividad de Bosques Alto Andinos (Polylepis) en la Cuenca Alta Del Río Pativilca. Pontificia Universidad Católica del Perú. Lima. Perú.

Ecoan - Asociación Ecosistemas Andinos. 2011. Plan Maestro de las Áreas De Conservación Privada "Mantanay" "Abra Málaga”, “Qhishuarani”. Cusco (20112016). Boletín Informativo.

Fjeldså, J. \& Kessler, M. 1996. Conserving the Biological Diversity of Polylepis Woodlands of the Highland of Peru and Bolivia. A Contribution to Sustainable Natural Resource Management in the Andes. NORDECO, Denmark, Copenhagen.

Galiano, W., Tupayachi, H., Condori., R. \& Choquehuanca., P. 2000. Revisión del Género Polylepis (Rosaceae), Para el Sur del Perú: Caso Bosques Altoandinos del Valle de Urubamba. Resúmenes del I Congreso Internacional de Ecología y Conservación de Bosques de Polylepis. CochabambaBolivia. 
Galiano, W. 1994. Los Bosques más Altos del Mundo: Base Fundamental para el Eco.desarrollo en los Andes. Rev. Opciones. UNSAAC - INANDES.27-35:1.

Gobierno Regional Cusco. 2012. Memoria descriptiva mapa de pendientes Región Cusco. Fortalecimiento del desarrollo de capacidades de ordenamiento territorial en la Región Cusco [Proyecto]. Área de Fisiografía. Gerencia regional de planeamiento, presupuesto y acondicionamiento territorial. Sub gerencia de acondicionamiento territorial: pp $15-$ 26. Cusco.

Inrena, 1995. Mapa Ecológico del Perú: Guía Explicativa. Ministerio de Agricultura. Lima-Peru.

Kessler, M. 2006. Bosques de Polylepis: Botánica Económica de los Andes Centrales. Ed. Moraes, M.; Ollgard, B.; Kvist, P.; Borchsenius, F. \& Balslev, H. Universidad Mayor de San Andrés, La Paz, Bolivia. pp 110-120.

Mendoza, W. \& Cano, A. 2011. Diversidad del Género Polylepis (Rosaceae, Sangusorbeae) en los Andes Peruanos. Rev. Peru. biol. 18(2): 197-200 (Agosto 2011). Facultad de Ciencias Biológicas UNMSM.

Ministerio de Agricultura. 1995. Mapa Ecológico del Perú. [Guía explicativa]. INRENA. Perú.

Pascual, L. \& Torné, J. 2009. Conefor Sensinode 2.2: a software package for quantifying the importance of hábitat patches for landscape connectivity: Enviromental Modelling \& Software 24, 135-139.

Romoleroux, K. 1992. Rosaceae in the Páramo of Ecuador. In Balslev H. \& J.L. Luteyn (eds.) Páramo: An Andean Ecosystem under Human Influence: 85-94.

Saura, S. 2009. Measuring connectivity in landscape networks: towards meaningful metrics and operational decision support tools. En: Catchpole, R., Smithers, R., Baarda, P., Eycott, A. (Eds), And ecological networks: science and practice. Proceedings of the 16th Annual IALE (UK) Conference.pp. 1-10.
Saura, S., Gonzales, A. \& Ramón, E. 2011. Evaluación en los cambios en la Conectividad de los bosques. El índice del área conexa equivalente y su aplicación a los bosques de Castilla y León. (1) Departamento de Economía y Gestión Forestales. ETSI. Montes. (2) Departamento de Silvopascicultura. ETSI. Forestal. Universidad Politécnica de Madrid. Ciudad Universitaria s/n. Madrid. España.p (01 - 22).

Saura, S. \& Rubio, L. 2009b. Conefor Sensinode, una nueva herramienta para el análisis de la conectividad de los bosques: fundamentos y experiencias de aplicación. Actas del V Congreso Forestal Español. Sociedad Española de Ciencias Forestales.

Schmidt-lebuhn, A., Kessler, M. \& Kumar, M. 2006. Promiscuity in the Andes: species relationships in Polylepis (Rosaceae, Sanguisorbeae) based on AFLP and morphology. Systematic Botany, 31(3): pp. 547-559.

Segura, M. \& Trincado, V. 2003. Cartografía digital de la Reserva Nacional Valdivia a partir de imágenes satelitales Landsat TM. Digital cartography of the National Reserve in Valdivia based on Landsat TM images. Bosque, Vol. $24 \mathrm{~N}^{\circ}$ 2, 2003, pp. 43-52. Laboratorio de Geomática, Instituto de Manejo Forestal, Facultad de Ciencias Forestales, Universidad Austral de Chile, Casilla 567, Valdivia, Chile.

Senamhi - Minam. 2012. Vigilancia de la Radiación Ultra Violeta- B En Ciudades Importantes Del País.Vol. 08.pp (01 - 03).

Servat, G., Mendoza, W. \& Ochoa, A. 2002. Flora y Fauna de cuatro especies de Polylepis (ROSACEAE) en la Cordillera delVilcanota (Cusco, Perú). Ecología Aplicada, diciembre, año/vol. 1, número 001.pp. 2535. Universidad Nacional Agraria La Molina. Lima, Perú.Perú. 
Simpson, B. 1979. A Revision of the genus Polylepis (Rosaceae: Sanguisorbeae) Smithsonian Contribution to botany; $\mathrm{n}^{\circ} 43$.

Troll, C. 1971. Landscape Ecology (Geoecology) and Biogeocenology - A terminological Study. Geoforum, $\mathrm{n}^{\circ} 8,43-46$.

Tupayachi, A. 2004. "Evaluación de los Bosques Altoandinos de Polylepis (Rosaceae) del Valle Sagrado de los Inkas, para una Propuesta de Area de Conservación Regional (ACR)". Maestria en Ciencias: Mención En EcologíaY Recursos Naturales. Escuela de PostGrado. UNSAAC- Cusco

Tupayachi, A. 2005. Flora de la Cordillera de Vilcanota.ARNALDOA 12 (1-2): 126 - 144 Área de Biología Vegetal. Facultad de Ciencias Biológicas. UNSAAC- Cusco.

Yallico, E. 1992. Distribución de Polylepis en el Sur de Puno. Proyecto Arbolandino. Apoyo al Desarrollo Forestal Comunal de la Región Altoandina. Puno, Perú

UICN. 2000. Categorías y criterios de la Lista Roja de la UICN versión 3.1 segunda edición. pp (05) (14 - 27). Comisión de Supervivencia de Especies. Gland. Suiza.

\section{Páginas web}

Climate $\mathrm{Nasa}$. Disponible en: http://climate.nasa.gov/state of flux\#Image sofchange.jpg Glovis. Disponible en: http://glovis.usgs.gov/ I $\mathrm{g} \mathrm{n}$ D is pon i b e e n : http://www.ign.gob.pe/?PG=Servicio_cartog rafia. 